\title{
Grand challenges in interdisciplinary physics
}

\author{
Alex Hansen * \\ Department of Physics, Institutt for fysikk, Norges Teknisk-Naturvitenskapelige Universitet, Trondheim, Norway \\ ${ }^{*}$ Correspondence: alex.hansen@ntnu.no \\ Edited by: \\ Christine Charles, The Australian National University, Australia \\ Reviewed by: \\ Ferenc Kun, University of Debrecen, Hungary \\ Christine Charles, The Australian National University, Australia
}

Keywords: interdisciplinary physics, computational physics, occam's razor, fluid dynamics, rheology, granular media

The dictionary definition of physics is the study of matter, energy, and the interaction between them ${ }^{1}$. There is no doubt that this definition does describe the core of physics. However, over the last three decades, a shift has occured. Physics has spilled over its boundaries set by the definition quoted, and we now have fields such as econophysics, sociophysics, biological physics, and geological physics. Common for these new fields of physics is that if they are to be defined based on the subjects that are studied, they would not belong to physics. What does make them part of physics is rather the way the subjects are studied. By regarding these new fields as belonging to physics, the dictionary definition of physics no longer holds. One has to revise it. It makes sense to replace the old definition stating that physics is the study of certain quantities by one where physics is defined as a method to approach scientific problems.

Physics as a method of approaching scientific problems is different from other sciences. It is hierarchical: more general questions are posed and answered before more specific questions. Here is a concrete example: In the twenties, general quantum mechanics was developed. In the thirties, a general theory of metals was developed. This allowed for studying specific metals, but it also opened up for the search for a class of materials that were between metals and insulators: the semiconductors. In the forties, this resulted in the construction of the first transistor-and the electronics age was born. One may only speculate how long it would have taken to construct

${ }^{1}$ See e.g., physics.org or www.thefreedictionary.org. the transistor if this path from the more general to the more specific had not been followed. How long would it take before someone accidentally stumbled across the semiconductor?

This approach sometimes attracts ridicule from people outside physics, especially engineers that have to solve specific problems within specific deadlines rather than finding the solution of a general problem and then work from there taking the time that is necessary. One well known story is that of the theoretical physicist who is asked to solve a specific problem in connection with agricultural milk production. He comes back after some time declaring that he has the solution, but only in the limit of spherically symmetric cows.

This hierarchical approach lies behind the extensive use of models in theoretical physics. For example, the Ising model for magnetic materials is a caricature of a real magnet. However, it does, also quantitatively, reproduce the phase diagram of a large class of magnets. The Potts model attracted critique when it was first published in 1952 [1] for being a model without physical contents. However, later on, it was understood that not only does it contain the Ising model as a special case, but it also describes a large number of other magnetic-and other physical systems beyond the Ising model [2] The Potts model is a good example of the use of physical models to study the physical phenomena of interest with the minimum of ingredients needed: these models are stripped of any irrelevant contents. In fact, the models, and the approach of physics to science, is related to Occam's dictum: Numquam ponenda est pluralitas sine necessitate [Plurality must never be posited without necessity] $[3]^{2}$.

Physics that focuses on problems outside its traditional boundaries belongs almost by definition to interdisciplinary physics. That physics spills over its traditional boundaries is a phenomenon that is just a few decades old. What lies behind this evolution? It is the advent of the computer as a serious research tool. We are not there yet, but computational physics is rapidly establishing itself as a third way of doing physics on equal footing with experimental and theoretical physics. The power of modern computer, being in the form of a huge machine such as the Japanese $\mathrm{K}$-computer consisting of 800 racks or in the form of GPUs-Graphic Processing Units-which allows enormous power on the desktop thanks to the computer game industry, allows for handling problems that would be forbidding even to think of in earlier times. In fact, the "Avogadro challenge" [5] - the ability to simulate macroscopic properties with microscopic models - and the Bluebrain project ${ }^{3}$ — the simulation of a virtual human brain-are now being approached rapidly with the emergence of petaflop computing. This has also opened up for entirely new concepts such as networks possessing a scalefree topology [6]. The computer will continue increasing its importance in physics and with it, physics will expand its basin of interest.

\footnotetext{
${ }^{2}$ Einstein is often quoted as having stated "Everything should be as simple as possible, but not simpler." This is of course the Occam razor [4].

${ }^{3}$ See e.g., bluebrain.epfl.ch
} 
Interdisciplinary physics as defined by our journal Frontiers in Physics collects under its umbrella:

- Statistical physics,

- Thermodynamics and non-linear systems,

- Fluid mechanics,

- Rheology and elasticity,

- Soft matter physics,

- Granular matter,

- Physics of fracture,

- Networks and network theory,

- Traffic and transport,

- Complex systems,

- Environmental physics,

- Energy sources (not including nuclear energy),

- Energy storage,

- Industrial and technological research,

and other fields that do not naturally fit within the other sections of the journal.

Let us consider fluid mechanics. The basic equations governing the flow of newtonian fluids, the Navier-Stokes equations, were in place some 170 years ago. From the moment they were written down, the field went from being a collection of experimental observations to being a problem of solving a set of differential equations with different boundary conditions. The intricacies of fully developed turbulence hides in the Navier-Stokes equations. Raw computer power makes it possible to get closer to this elusive problem, but still our understanding of the phenomenon is more qualitative than quantitative.

The dynamics of interacting vortex strings is related to turbulence. When the strings entangle and stretch they presumably form the path toward fully developed turbulence. In step with the growing computer power, we understand more and more of this extremely complex phenomenon of great practical importance.

Of course it is not precise to claim that fluid mechanics is just the solution of a set of differential equations. Cavitation phenomena requires coupling to other fields such as thermodynamics.
From a practical point of view, cavitation is a source of noise pollution, e.g., from airplane engines besides corroding the materials that form the boundaries of the cavitating fluid. The still mysterious phenomenon of sonoluminescence where an oscillating bubble is formed in a liquid when sound waves are focused at a given point in it, resulting in the emmission of light [7], is a form of cavitation. What is its explanation?

When the fluids no longer are newtonian, we enter the realm of rheology. Fluids such as ketchup are shear-thinning. That is, the larger the shear force, the easier they flow. Shear-thickening fluids behave in the opposite way. Such a fluid is easily made in the kitchen by forming a high-concentration suspension of corn starch in water. Such fluids are challenging to model. They may be mathematically modeled as Herchel-Bulkley fluids [8] whose viscosity is shear rate dependent. However, they are computationally very challenging, even under seemingly simple conditions. Again, important practical processes depend on a deep understanding of such fluids. A very current example is fracking where shales are fractured using water-based slurries that are highly non-newtonian. Why? They need to keep the fractures open once they have been created.

Moving further down the list, we come to granular media. Sand is a good example. Sand may flow as a fluids, behave as a gas or-when left alone-act as a solid. However, sand is neither of them. The equations governing gases, fluids or solids do not provide reliable descriptions of the phases. In fact, the physics of granular media is where the physics of fluids were before the advent of the Navier-Stokes equations: the field essentially consists of a collection of phenomena that each has been described to a varying degree of precision, but there is no theory binding them together. When considering that a non-negligible percentage of the world's energy consumption is spent handling powders, this is indeed a sorry state of matter. But, progress is being made. It is a very active field of research.

This was just three of the fields that belong to interdisciplinary physics. The other fields all possess challenging problems that are of significant relevance. In the fields driven by large computer power, one must expect that progress will be in step with the growth of the computer. In the other fields, it will just be cleverness and inventiveness that will drive us forwards.

\section{REFERENCES}

1. Potts RB. Some generalized order-disorder transformations. Math Proc. (1952) 48:106. doi: 10.1017/S0305004100027419

2. Wu FY. The potts model. Rev Mod Phys. (1982) 54:235. doi: 10.1103/RevModPhys.54.235

3. Soklakov AN. Occam's razor as a formal basis for physical theory. Found. Phys. Lett. (2002) 15:107. doi: 10.1023/A:1020994407185

4. Sessions R. How a Difficult Composer Gets That Way. New York, NY: ProQuest (1950).

5. Ito N. "The Avogadro challenge," in Computer Simulation Studies in Condensed Matter Physics XIX. Springer Proceedings in Physics, Vol. 123. Landau DP, Lewis SP, Schöttler HB, editors. Berlin: Springer (2009). 48-55.

6. Albert R, Barabási AL. Statistical mechanics of complex networks. Rev Mod Phys. (2002) 74:47. doi: 10.1103/RevModPhys.74.47

7. Brenner MP, Hilgenfeld S, and Lohse D. SingleBubble sonoluminiscence. Rev Mod Phys. (2002) 74:425. doi: 10.1103/RevModPhys. 74.425

8. Herschel WH, Bulkley R. Konsistenzmessungen von gummi-bensollösungen. Koll. Zeitschr. (1926) 39:291

Conflict of Interest Statement: The author declares that the research was conducted in the absence of any commercial or financial relationships that could be construed as a potential conflict of interest.

Received: 22 August 2014; accepted: 23 September 2014; published online: 09 October 2014.

Citation: Hansen A (2014) Grand challenges in interdisciplinary physics. Front. Phys. 2:58. doi: 10.3389/ fphy.2014.00058

This article was submitted to Interdisciplinary Physics, a section of the journal Frontiers in Physics.

Copyright (c) 2014 Hansen. This is an open-access article distributed under the terms of the Creative Commons Attribution License (CC BY). The use, distribution or reproduction in other forums is permitted, provided the original author(s) or licensor are credited and that the original publication in this journal is cited, in accordance with accepted academic practice. No use, distribution or reproduction is permitted which does not comply with these terms. 